\title{
SPECTRAL MODELLING OF FORCE RIPPLES IN PERMANENT MAGNET BEARINGLESS MOTORS
}

\author{
B. Lapôtre ${ }^{1}$, N. Takorabet ${ }^{1}$, F. Meibody-Tabar ${ }^{1}$, R. Lateb ${ }^{2}$, J. Dasilva ${ }^{2}$ \\ Université de Lorraine, GREEN-ENSEM, 2 avenue de la forêt de Haye 54518 Vandoeuvre lès Nancy - France \\ SKF Magnetic Mechatronics, 2 rue des champs, 27250 Saint Marcel, France \\ noureddine.takorabet@univ-lorraine.fr
}

\begin{abstract}
Purpose - This paper proposes an analytical model for the calculation of forces ripples in bearingless permanent magnet motor. density

Design/Methodology/Approach - The model is based on a spectral modeling of the forces based on a spectral analysis of the air-gap flux

Finding - The proposed methodology allow the prediction of the ripples of the levitation according the design of the machine and the topology of the winding. It can be used in the design procedure of bearingless motor in order to avoid mechanical resonance of the rotor shaft.

Research limitations implications - This model cannot be used for high saturated machines.

Originality/Value - The main originality of this paper consists on the evaluation of the field harmonics according to their sources and the contribution of the different harmonics to the force ripples. This can help the designer to focus on reducing or eliminating only the harmonics of flux density which create the force ripples.
\end{abstract}

Keywords - Magnetic levitation, Bearingless Motor, PM motor, High speed motors, Radial forces

1

\section{Introduction}

Nowadays, high speed or high purity applications are various in many industrial processes which need the use of magnetic bearings for electric machines. Topologies of electric motors with magnetic bearings are various, but the general principle of such machines is to use two radial bearings to control the position in the plane and one axial bearing to control the movement in the axial direction (Fig.1-a). To ensure the magnetic levitation the rotor must be magnetically stabilized in five degrees of freedom (3 translational and 2 tilting). The three magnetic bearings presented in Fig.1-a, ensure this stabilization. However, this makes the axial length of the shaft rather long and may increasing the power supplies of the machine. Increasing the axial length of the rotor leads to decrease the critical speed and mechanical frequencies.

Bearingless motors are electromagnetic devices that can generate both suspension force and torque in a single unit. They allow the suppression of one radial bearing as shown on Fig. 1-b. By the way, the axial length of the rotating shaft can be reduced and the critical speed increased. This solution reduces the number of power supplies but increase the complexity of the motor and requires a detailed modeling of the various interactions that may occur in the air-gap of the motor (Schneider 2007), (Oshima, 1996).

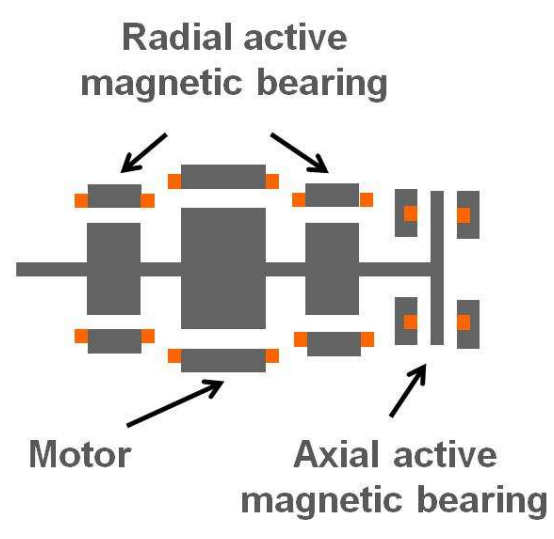

(a)

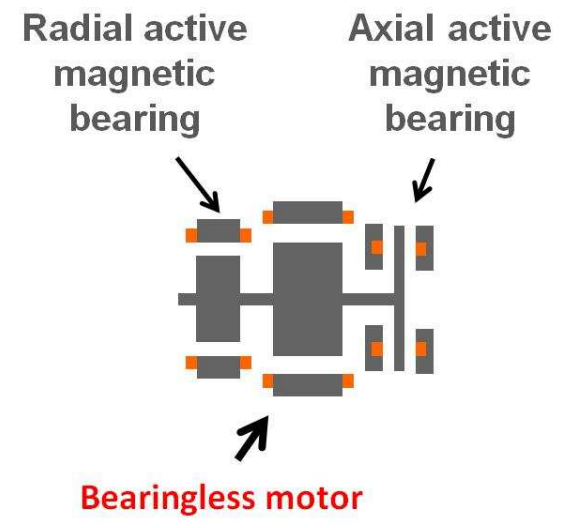

(b)

Fig. 1. From the magnetic bearing motor to a bearingless motor a- Classical High speed motor with 3 active magnetic bearings $\mathrm{b}$ - Bearingless motor with 2 active magnetic bearings

${ }^{1}$ The paper has been presented at the CISTEM conference 
Various topologies of bearingless motors have been proposed in the last decade. Many contributions dealed with PM motor, reluctance machine (Liu, 2009), induction motor (Chiba, 1997), or slotless disc motor (Steinert, 2013).

The knowledge of the harmonic content of the air-gap flux density allows the prediction of the force waveforms and its harmonic content. In this paper, an analytical model based on the spectral decomposition of the fields created by different sources (stator winding, rotor magnets, ...) is developed. Using a spectral approach, the contributions of different interactions between the harmonics of the radial and tangential components of the air-gap flux density are evidenced. Experimental tests are carried out on a prototype of bearingless motor designed and manufactured for this purpose.

\section{General thoery of multi-phase bearingless motors}

\subsection{Magnetic field approch}

The distribution of the flux density and magnetic field in the air-gap of electrical machines is essential in the process of energy conversion. Both stator and rotor armatures generate magnetic field in the air-gap. The calculation of the radial force needs the use the projection of the local magnetic stress on a given system of axes. This is suitable for the computations of the excitation forces for the mechanical modes. In magnetic levitation, the control of the first mode is required to control the forces in the $\mathrm{X}$ and/or $\mathrm{Y}$ directions. Forces along these axes can be expressed by:

$$
\begin{aligned}
& F_{x}=\frac{R L_{z}}{2 \mu_{0}} \int_{0}^{2 \pi}\left[\left(B_{n}^{2}-B_{t}^{2}\right) \cos \theta-2 B_{t} B_{n} \sin \theta\right] d \theta \\
& F_{y}=\frac{R L_{z}}{2 \mu_{0}} \int_{0}^{2 \pi}\left[\left(B_{n}^{2}-B_{t}^{2}\right) \sin \theta+2 B_{t} B_{n} \cos \theta\right] d \theta
\end{aligned}
$$

where $B_{n}$ and $B_{t}$ are respectively the normal and tangential component of the flux density. $R$ is the radius of a circular path $\Gamma$ shown on Fig. $2 . L_{z}$ is the length of the iron stack, $\theta$ is angular position and $\mu_{0}$ the permeability of vacuum.

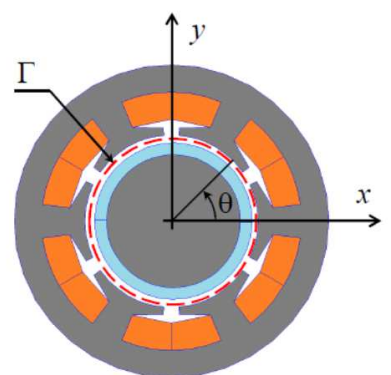

Fig. 2. Schematic representation of the bearingless motor: $\mathrm{XY}$ axes, Path for Tensor calculation, $\theta$ is the position angle.

\subsection{Multi-phase electric systems approch}

In order to control the space harmonic content of air-gap flux density, with multiphase machines, the use of general theory of multi-phase systems is required. Let's consider a $q$-phase winding with $q$ concentric coils. This winding can be supplied in order to create a $p$-pole flux density in the air-gap. The generalized Concordia transformation can be applied to obtain different two-phase equivalent systems.

$$
\left[i_{h 1} i_{\alpha 1} i_{\beta 1} i_{\alpha 2} i_{\beta 2} \ldots .\right]=T_{q q}^{t}\left[i_{1} i_{2} i_{3} \ldots . i_{q}\right]
$$

where $T_{q q}^{t}$ is the gerneralized Concordia transformation matrix of order $q$.

The equivalent currents $i_{\alpha 1}, i_{\beta 1}$ represent the "main" 2-phase system denoted by $I_{\alpha \beta 1}$ and create an equivalent current sheet with $p$ poles on the inner surface of the stator. The currents $i_{\alpha 2}, i_{\beta 2}$ represent the first "secondary" system denoted by $I_{\alpha \beta 2}$. It is characterized by $2 p$ poles. If the number of stator phases is greater than 6 the generalized Concordia transformation generates additional "secondary" systems denoted by $I_{\alpha \beta m}$ where $m$ is the system order which cannot exceed $((q-1) / 2)($ Semail, 2004). 
In the case of bearingless motor, it is necessary to create two equivalent current sheets of 2 and 4 poles respectively in order to ensure the levitation force. Therefore, if the pole number of the main system $I_{\alpha \beta 1}$ is $p=1$, the pole number of the secondary system $I_{\alpha \beta 2}$ is: $2 p=2=p+1$. So the currents $I_{\alpha \beta 1}$ and $I_{\alpha \beta 2}$ have to be controlled carefully to generate force and torque with the same multi-phase system.

From the magnetic field approach, the equivalent systems $I_{\alpha \beta 1}$ and $I_{\alpha \beta 2}$ create two current sheets in the inner surface of the stator that could be created by two separate 3-phase windings. The first one is of 2 poles while the second one has 4 poles. The difference between the two approaches lies with the electric connectivity of the phases and the power supply. Hereafter, the analysis will carry on with the principle of generalized multi-phase theory even if the study can be restricted to the case of two 3-phase winding which is a special and particular case.

The multiphase windings generate high order harmonics and their ranks depend on the pole number and phase number. These harmonics can be significant and have to be taken into account and in order to consider their contribution to the torque and levitation force. The "main" system $I_{\alpha \beta 1}$ generates harmonics of order: $m q \pm p$ where $m$ is a given integer whereas the system $I_{\alpha \beta 2}$, generates harmonics of order : $m q \pm 2 p$. As examples, we consider the cases of 5-phase and 6-phase motors with 2 poles $(p=1)$, the stator winding generates harmonics of the flux density presented in Tab. I where the nature of the corresponding waves is specified by "F" for forward harmonics and "B" for backward harmonics.

On the other hand, if we take a look on the field harmonics generated by the rotor, they depend on the topology of the rotor (surface mounted, surface inserted, buried, ...). For surface magnet rotors, we can identify two cases: Parallel magnetization or Radial magnetization of the magnets. In the first case the air-gap field is sinusoidal and only one harmonic exists while in the second case all odd harmonics are in presence. All these harmonics are forward because they are moved by the rotor in the positive direction (Tab. I). Interactions of these harmonics must be taken into account in the calculation of the force in order to control the levitation force and electromagnetic torque.

Table 1: Magnetomotive Force (MMF) harmonic representation due to the systems $I_{\alpha \beta 1} \quad I_{\alpha \beta 2}$ and the magnets - 5-phase winding

\begin{tabular}{|c|c|c|c|c|c|c|c|c|c|c|c|c|}
\cline { 2 - 12 } \multicolumn{1}{c|}{} & \multicolumn{10}{c|}{ MMF Harmonics } \\
\cline { 2 - 12 } \multicolumn{1}{c|}{} & 1 & 2 & 3 & 4 & 5 & 6 & 7 & 8 & 9 & 10 & 11 & 12 \\
\hline$I_{\alpha \beta 1}$ & $\mathrm{~F}$ & & & & $\mathrm{~B}$ & & $\mathrm{~F}$ & & & & $\mathrm{~B}$ & \\
\hline$I_{\alpha \beta 2}$ & & $\mathrm{~F}$ & & $\mathrm{~B}$ & & & & $\mathrm{~F}$ & & $\mathrm{~B}$ & & \\
\hline
\end{tabular}

\begin{tabular}{|c|c|c|c|c|c|c|c|c|c|c|c|}
\cline { 2 - 12 } \multicolumn{1}{c|}{} & \multicolumn{10}{c|}{ Permanent magnet Harmonics } \\
\cline { 2 - 12 } \multicolumn{1}{c|}{} & 1 & 2 & 3 & 4 & 5 & 6 & 7 & 8 & 9 & 10 & 11 \\
\hline Parallel & $\mathrm{F}$ & & & & & & & & & & \\
\hline Radial & $\mathrm{F}$ & & $\mathrm{F}$ & & $\mathrm{F}$ & & $\mathrm{F}$ & & $\mathrm{F}$ & & $\mathrm{F}$ \\
\hline
\end{tabular}

\section{Spectral analysis of the suspention force}

The case of smooth rotor is considered. The calculation of the air-gap field distribution can be performed by either analytical or numerical model. The model developed in this paper begins with the spectral analysis of the magnetic flux density over a circular path $\Gamma$ passing through the air-gap for each source of flux density. By neglecting the saturation of the iron core, the superposition principle can be applied. The normal and tangential components of the flux density for each sources are expressed as follows (Lapôtre, 2014):

$$
\left\{\begin{array}{l}
B_{n}(\theta)=\frac{1}{2} \sum_{k, S}\left|B n_{s, k}\right| e^{j \theta_{n_{-}, k, k}} e^{j k \theta}+\left|B n_{s, k}\right| e^{-j \theta_{n_{-}, k, k}} e^{-j k \theta} \\
B_{t}(\theta)=\frac{1}{2} \sum_{k, s}\left|B t_{s, k}\right| e^{j \theta_{t_{-}, k}} e^{j k \theta}+\left|B t_{s, k}\right| e^{-j \theta_{t_{-}, k}} e^{-j k \theta}
\end{array}\right.
$$

In this equation $\left|B n_{s, k}\right|$ and $\left|B t_{s, k}\right|$ represent the amplitudes of the radial and tangential components of the flux density due to the source "s". The angles $\theta_{n_{-} s, k}$ and $\theta_{t_{-} s, k}$ are their respective phases. The subscript " $s$ " corresponds to the different field sources which exist in the motor. In the studied motor we have the two systems $I_{\alpha \beta 1}, I_{\alpha \beta 2}$ and the rotor magnets.

The use of the force components and spectral decomposition of the air-gap flux density, allows establishing accurately the harmonics contributions to the levitation force. The combination of equations (1) and (3) allows determining the components $F_{x}$ and $F_{y}$ which can then be expressed by: 


$$
\left\{\begin{array}{l}
F_{x}=\frac{R L_{Z} \pi}{2 \mu_{0}} \sum_{s, a, k} F X_{k, s, a} \\
F_{y}=\frac{r \cdot L_{Z} \pi}{2 \mu_{0}} \sum_{s, a, k} F Y_{k, s, a}
\end{array}\right.
$$

Where the components $F X_{k, s, a}$ and $F Y_{k, s, a}$ are the elementary forces generated by the interaction of two successive harmonics of rank $k$ and $k+1$ due to similar or different sources. Considering the phase shift of normal and tangential flux density harmonics according to the source location, the force $F X_{i, s \sigma}$ and $F Y_{i, s \sigma}$ can be simplified as follows (Lapôtre 2014):

$$
\begin{aligned}
F X_{i, s, a} & =K \cos \left(\theta_{n_{-}, i}-\theta_{n_{\_} a, i+1}\right) \\
F Y_{i, s, a} & =K \sin \left(\theta_{n_{-}, i}-\theta_{n_{\_} a, i+1}\right)
\end{aligned}
$$

Therefore the subscripts $s$ and $\sigma$ can have the values "out" in the case of outside source (winding) and "in" in the case of inside source (magnets). The amplitude $K$ depends on the location of the sources relative to the contour. The four possible cases are summarized in Tab. II.

Table 2: Expression of the amplitude $\mathrm{K}$ according to the location of the sources

\begin{tabular}{ccc}
\hline & $B_{i, o u t}$ & $B_{i, i n}$ \\
\hline$B_{i+1,0 u t}$ & $\left(\left|B_{n a, i+1}\right|+\left|B_{t a, i+1}\right|\right) \cdot\left(\left|B_{n s, i}\right|-\left|B_{t s, i}\right|\right)$ & $\left(\left|B_{n a, i+1}\right|+\left|B_{t a, i+1}\right|\right) \cdot\left(\left|B_{n s, i}\right|+\left|B_{t s, i}\right|\right)$ \\
$B_{i+1, i n}$ & $\left(\left|B_{n a, i+1}\right|-\left|B_{t a, i+1}\right|\right) \cdot\left(\left|B_{n s, i}\right|-\left|B_{t s, i}\right|\right)$ & $\left(\left|B_{n a, i+1}\right|-\left|B_{t a, i+1}\right|\right) \cdot\left(\left|B_{n s, i}\right|+\left|B_{t s, i}\right|\right)$ \\
\hline
\end{tabular}

In this table, the magnitude $K$ is determined by the sum or the difference of positive terms according to the harmonic rank and the location of the sources ("in" or "out"). Using the expressions given in this table, the harmonic content of the flux density can be chosen in order to maximize the fundamental component of levitation force and to minimize the harmonic content as well. Illustrative examples will be given in the following.

\section{Application to 6-phase Windings}

The developed model allows determining the levitation force for any topology and takes into account the space harmonics of the magnetic field and the corresponding force ripples. In this section, the case of 6-phase with radial or parallel magnetized rotor are presented and discussed. For each case, the force waveforms acting on the rotor over a whole period is determined by the proposed model as well as by full FEM computation. To see the influence of high order space harmonics, the currents are controlled in order to generate a constant levitation force. The influence of the high order harmonics is highlighted.

Consider a 6-phase, 2-pole permanent magnet machine. The stator winding composed by 6 concentrated coils which can be supplied by a 6-leg inverter. The stator winding is supplied by the superposition of two 6-phases currents corresponding to the systems $I_{\alpha \beta 1}$ and $I_{\alpha \beta 2}$ :

$$
\left\{\begin{array}{c}
i_{i, 1}=I_{\alpha \beta 1} \cos \left(\omega_{1} t+\phi_{1}+\frac{(i-1) \pi}{6}\right) \\
i_{i, 2}=I_{\alpha \beta 2} \cos \left(\omega_{2} t+\phi_{2}+\frac{2(i-1) \pi}{6}\right)
\end{array}\right.
$$

$I_{\alpha \beta 1}, I_{\alpha \beta 2}, \omega_{1}, \omega_{2}, \phi_{1}$, and $\phi_{2}$ represent the amplitudes, frequencies and the phases of the two systems respectively. The currents $I_{\alpha \beta 1}$ generate a 2-pole magnetomotive force (mmf) in the air-gap and the currents $I_{\alpha \beta 2}$ generate a 4-pole mmf. According to the multiphase winding theory, the two systems generate forward harmonics of the air-gap flux density of rank $6 m+1$ and $6 m+2$ and backward harmonics of rank $6 m-1$ and $6 m-2$. These travelling waves have different mechanical velocities but they present the electrical frequencies $\omega_{1}, \omega_{2}$, when they are expressed in a fixe frame.

In the case of parallel magnetized rotor, the magnets generate a pure sinusoidal flux density with the frequency $\omega$ due to the rotor speed. All these existing harmonics and their frequencies are summarized in Tab. III. The harmonic interactions and pulsations in the case of radial magnetized rotor are given in Tab. IV.

In these tables the different pairs of harmonics which interact and generate radial force components are evidenced. They can be gathered in three groups highlighted with the three colors (red, blue, yellow). They correspond to the different cases listed in Tab. II:

- red: force component which norm is the product of two sums,

- blue: forces component which norm is the product of two subtractions,

- yellow: forces which norm is product of subtraction and sum. 
In the case of parallel magnetized rotor, the field due to the magnets is sinusoidal. Only the first harmonic exists. The forces of the second group (blue) do not exist. They appear if the rotor magnets are radial magnetized.

\subsection{Case of parallel magnetized rotor:}

This table allows highlighting the interactions between the field harmonics. The general form of the elementary force due to a couple of field harmonics is of the form:

$$
F_{i}=K \cos \left(\omega_{1}-\omega_{2}+\phi\right)
$$

where $\phi$ is the shift phase between the two filed harmonics.

It is remembered that to have a constant torque, the electric pulsation of the $I_{1}$ system and the mechanical pulsation must be equal $\omega=\omega_{1}$. In order to obtain a constant levitation force it is necessary to have $\omega_{1}=\omega_{2}$. Figure 3-a. shows the force components $F_{x}$ and $F_{y}$ obtained by the proposed analytical model and by a full FEM computation. There is an excellent accordance between the two models and the curves are coincident.

Table 3: Harmonics pulsations and interactions for 6 phase parallel magnetized rotor

\begin{tabular}{|c|c|c|c|c|c|c|c|c|c|}
\hline \multirow{2}{*}{$\begin{array}{c}\text { Root } \\
\text { field }\end{array}$} & \multicolumn{7}{|c|}{ Space harmonium } \\
\cline { 2 - 10 } & 1 & 2 & 3 & 4 & 5 & 6 & 7 & 8 & 9 \\
\hline I1 & $\omega_{1}$ & & & & $-\omega_{1}$ & & $\omega_{1}$ & \\
\hline I2 & & $\omega_{2}$ & & $-\omega_{2}$ & & & & $\omega_{2}$ & \\
\hline Magnet & $\omega$ & & & & & & & \\
\hline
\end{tabular}

Table 4: Harmonics pulsation and interactions for 6 phase radial magnetized rotor

\begin{tabular}{|c|c|c|c|c|c|c|c|c|c|}
\hline \multirow{2}{*}{$\begin{array}{l}\text { Root } \\
\text { field }\end{array}$} & \multicolumn{9}{|c|}{ Space harmonium } \\
\hline & 1 & 2 & 3 & 4 & 5 & 6 & 7 & 8 & 9 \\
\hline I1 & $\omega_{1}$ & & & & $-\omega_{1}$ & & $\omega_{1}$ & & \\
\hline $\mathrm{I} 2$ & & & & & & & & & \\
\hline Magnet & & & & & $5 \omega$ & & $7 \omega$ & & \\
\hline
\end{tabular}

\subsection{Case of radial magnetized rotor :}

Radial magnetized rotor generates flux density in the air-gap with odd space harmonics of rank $2 m+1$ and frequencies $(2 m+1) \omega$. These harmonics interact with the field harmonics due to the current system $I_{\alpha \beta 2}$ which are show in red and blue in Tab.III. The general form of the elementary force due to a couple of field harmonics is of the form:

$$
F_{i}=K \cos \left(\mp(2 m+1) \omega \mp \omega_{2}+\phi\right)
$$

According to the results given above the mechanical pulsation and the electrical pulsation of the current system $I_{\alpha \beta 2}$ must be equal. In this condition, the levitation force generated by their interaction has a constant component as well as ripples of frequency: $2 m \omega$. On Figure 3-b, we show the $F_{x}$ and $F_{y}$ component of the levitation force obtained in this case by using both analytical model and full FEM computations. It can be seen that there is an excellent agreement between the two models once again.

The spectral analysis of the forces waveforms is shown on Fig. 4 for both $F_{x}$ and $F_{y}$ components. This spectral analysis allows the identification of the interaction between the different harmonics of the flux density. For example, the interaction of the 2 nd field harmonic due to the currents system $I_{2}$ and the 3 rd field harmonic due to the magnets produces the 2nd harmonic of the force. Henceforth, the following notation $\left(2_{I_{\alpha \beta 2}}, 3_{\text {mag }} \Rightarrow 2_{\text {force }}\right)$ will be adopted to describe such situation.
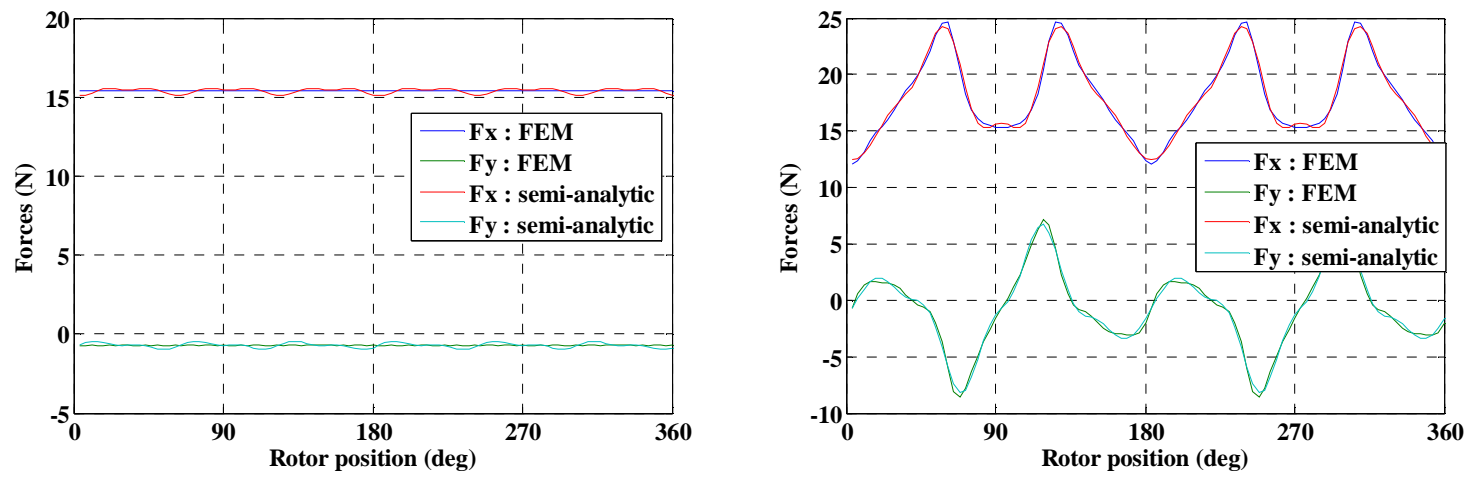
Fig. 3: Forces wavforms : (b) Six phase motor with parallel magnetization, (a) Six phase motor with radial magnetization

The interaction $\left(4_{I_{\alpha \beta 2}}, 3_{\text {mag }} \Rightarrow 4_{\text {force }}\right)$ corresponds to the red group characterized by the product of two sums while the interaction $\left(2_{I_{\alpha \beta 2}}, 3_{\text {mag }} \Rightarrow 2_{\text {force }}\right)$ corresponds to the blue group characterized by the product of two subtractions. That's why that the amplitude of the 4 th harmonic of the force is higher than the amplitude of the 2 nd harmonic.

Furthermore, it can be seen that the harmonics of higher ranks may have several origins. For example the interactions $\left(4_{I_{\alpha \beta 2}}, 5_{m a g} \Rightarrow 6_{\text {force }}\right)$ and $\left(8_{I \alpha \beta_{2}}, 7_{\text {mag }} \Rightarrow 6_{\text {force }}\right)$ lead to the 6 th harmonic of force. These harmonics are weak because they are generated by high rank field harmonics of low amplitude.

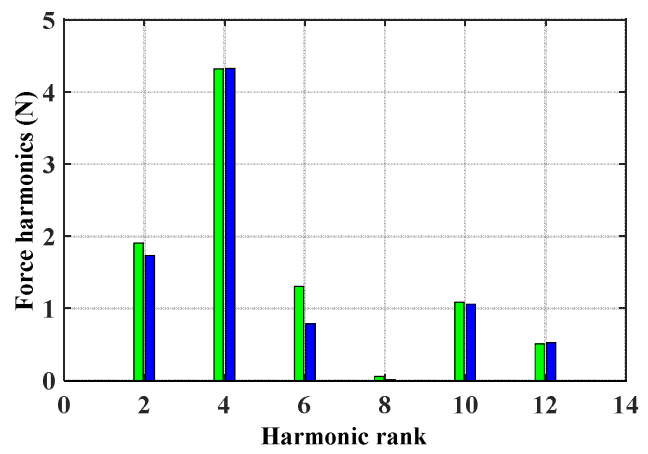

Fig. 4 : 6-phase motor -force harmonic analysis for radial magnetization

\section{Experimental results}

The experimental tests are carried out on a prototype that is designed and manufactured for this specific purpose. The speed rise of the machine is up to $60000 \mathrm{rpm}$ and the power rate is about few hundred Watts. Regardless the numerical data of this machines, the general topology of the prototype is presented hereafter. The target of these tests consist in the validation of the principle of two 3-phase separate windings and the analysis of the force harmonic content.

The stator has 6 slots and two double layer concentrated windings. The "torque" winding is designed to create a 2-pole flux density while the "force" winding creates a 4-pole flux density in the air-gap. The rotor is made up with a smooth armature with a magnet ring with parallel magnetization. It is made up with plastic bounded magnets.

Even if the machine is designed as a bearingless motor, the two active radial magnetic bearings are conserved as well as the axial one. The radial bearings can be used as sensors of the force for the experiments and/or external excitation forces for the simulation of external perturbations (shocks). The active magnetic bearings are well identified and tested by the standards of SKF-S2M company. The general structure and a picture of the prototype are shown on Fig. 5. The system is driven with a dSPACE plateform and Matlab-Simulink. A general view of the test bench is shown on Fig. 6.


Fig. 5 : General architecture of the prototype of bearingless motor

The first tests consist in the force-current identification along the two axes at standstill and for different values of the rotor speed. The linear shape of the curves $F_{x}\left(I_{2}\right)$ and $F_{y}\left(I_{2}\right)$ is observed as predicted by the analytic model. As an illustration, the force-current curves at $\Omega=1000 \mathrm{rad} / \mathrm{s}(9550 \mathrm{rpm})$ are shown on Fig. 7. The experimental results show the linearity of these curves with the current as predicted by the simulation. However there is a slight shift between $F_{x}\left(I_{2}\right)$ and $F_{y}\left(I_{2}\right)$ in the experimental tests, probably 
due the offset in the measurement or mechanical unbalance. The presence of high level of harmonics of the force may lead to a larger difference on the two axes especially for low values of the rotor speed.



Fig. 6 : General architecture of the prototype ob bearingless motor
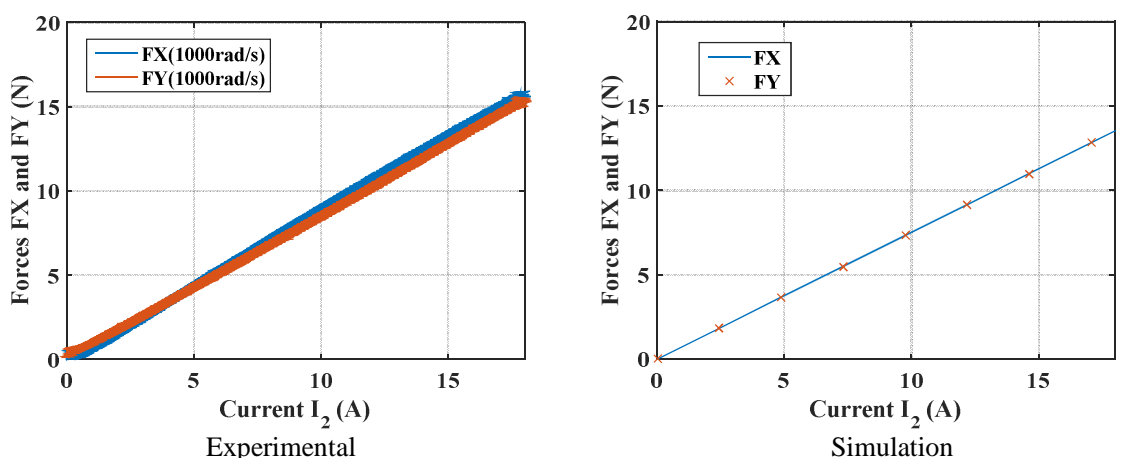

Fig.7 : Force-current characteristics

In a second step, the force waveforms are measured for different values of the rotor speed. The results are of best quality if the rotor speed is low. Indeed high speeds of the rotor lead to high frequencies of the force ripples. The response of the shaft may filter theses frequencies and the measurement is of bad quality. For a better precision of the measurement, it is recommended to perform the test for low speeds. On Fig. 8-a, the forces waveforms are shown in the case of $\Omega=300 \mathrm{rpm}$. The harmonics of the force are clear and their spectral representation is given on Fig. 8-b and a zoom is focused on the harmonics on Fig. 8-c. It can be clearly seen that only the 2 nd and 4th harmonics are significant on this figures for both $F_{x}$ and $F_{y}$. Theses harmonics are due the following interactions:

The 2 nd harmonic is due to the interaction : $2_{I_{\alpha \beta 2}}, 3_{\text {mag }} \Rightarrow 2_{\text {force }}$

The 4 th harmonic is due to the interaction : $4_{I_{\alpha \beta 2}}, 3_{\text {mag }} \Rightarrow 4_{\text {force }}$

The ratio between these two force harmonics is in agreement with the theoretical predictions given in Fig. 4.

Another important results that we can highlight is that the 4th harmonic which is due to high order and low amplitude harmonics of the flux densities is higher than the 2 nd harmonic of the force which is due to lower order and higher amplitude harmonics. This is due to the nature of the interactions predicted in Tab. IV. The 4th harmonic of the force is a "yellow" force due to the product of 2 subtractions while the 2nd harmonic is a "red" force with the product of two sums. Even if the amplitudes of the force harmonics are low, the right prediction of their presence and their importance is a good information for the supply control and the knowledge of the vibration of the rotor shaft. 


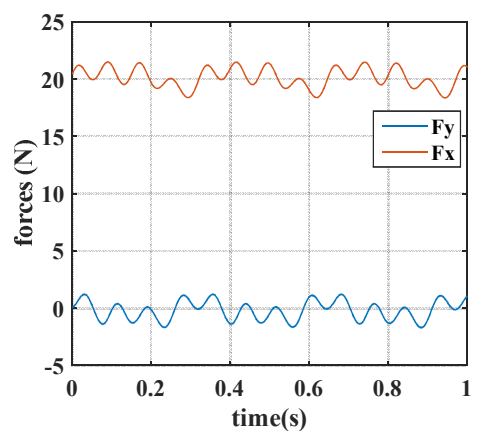

(a)

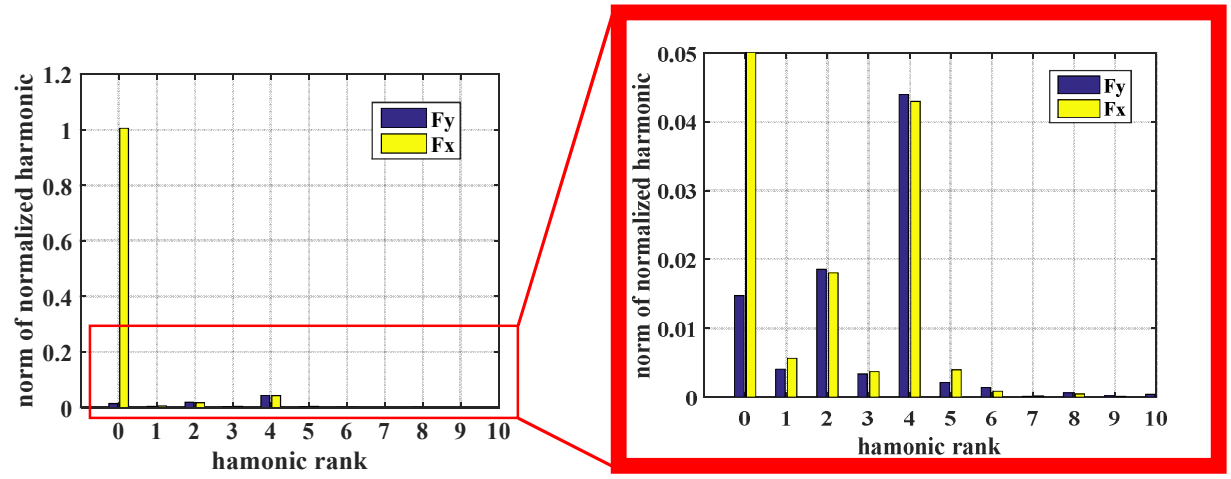

(b) (c)

Fig. 8 : Force waveforms and spectral analysis

\section{Conclusion}

This paper deals with the modeling of the force ripples of a bearingless motor. An analytical model based on the spectral decomposition of the air-gap flux density component is developed. The model is validated by full finite element model in many operating cases. A special focus is made on the harmonic content of the levitation force and their possible consequences. A special prototype is designed and manufactured for this purpose. Experimental tests confirm the theoretic prediction about the behavior of the force in terms of amplitude (average value) and harmonic content.

\section{References}

Schneider T. and Binder A., (2007), "Design and Evaluation of a 60000 rpm Permanent Magnet Bearingless High Speed Motor", IEEE PEDS'07, Bangkok, 2007

Oshima M., Miyazawa, S. Deido, T. Chiba, A. Nakamura, S. Fukao T., (1996), "Charactreristics of a permanent magnet type bearingless motor", IEEE trasactions on industry application, vol. 32, no. 2, pp. 363-370, 1996.

Liu Z., Deng Z., Cai J., Wu Y. and Wang, S. (2009), "Optimal design of a bearingless switched reluctance motor", Int. Conf. Applied Superconductivity and Electromagnetic Devices ASEMD,25-27 sept. 2009, Chengdu, pp. 241-245

Chiba A., Furuichi R., Aikawa Y., Shimada K., Takamoto Y., and Fukao T., (1997), "Stable operation of induction-type bearingless motors under loaded conditions", Industry Applications, IEEE Transactions on, vol. 33, ISSN : 0093-9994 , Jul/Aug 1997.

Steinert D., Nussbaumer T. and Kolar J. , (2013), "Concept of a $150 \mathrm{krpm}$ bearingless slotless disc drive with combined windings", Proceedings on Electric Machines \& Drives Conference (IEMDC) , pp. 311-318, 2013

Okada Y., Miyamoto S. and Ohishi T., (1996), " Levitation and Torque Control of Internal Perimanent Magnet Type Bearingless Motor", IEEE transactions on control systems technology, vol 4, no. 5, pp. 565-571, 1996.

Tiegna H., Bellara A., Amara Y. and Barakat G., (2012) "Analytical Modeling of the Open-Circuit Magnetic Field in Axial Flux Permanent Magnet Machines with semi-closed slots", IEEE Trans. Magn., vol. 48, no. 3, pp. 1212-1226, March. 2012.

Cao X., Deng Z., Yang G. and Wang, X. (2009), "Independent Control of Average Torque and Radial Force in Bearingless SwitchedReluctance Motors With Hybrid Excitations", Power Electronics, IEEE Transactions on, vol. 5, ISSN: 0885-8993, 2009.

Okada Y., Miyamoto S. and Ohishi, T., (1996), "Levitation and torque control of internal permanent magnet type bearingless motor", IEEE Transaction on control systems technology, vol. 4, no. 5, pp. 565-571, 1996.

Semail E., Kestelyn X. and Bouscayrol A., (2004), "Right harmonic spectrum for back-electromotive force of a n-phase synchronous motor", IEEE Conf. Indust. App., 2004

Lapotre B., Takorabet N., Meilbody-Tabar F., Lateb R. and Dasilva J., (2014), "On the use of spectral analysis of air-gap flux density in permanent magnet bearingless motors", Electrical Machines (ICEM), 2014 International Conference on, September 2014, Berlin, pp. 591-597.

Lapotre B., Takorabet N., Meibody-Tabar F., Fontchastagner J., Lateb R., Dasilva J., (2015), "New Model of Radial Force Determination in Bearingless Motor ", Magnetics, IEEE Transactions on, vol. 51, no. 3, 2015. 